\title{
Results on the use of tungsten heavy alloys in the divertor of ASDEX Upgrade
}

\author{
R. Neu ${ }^{\mathrm{a}, \mathrm{b}}$, H. Maier ${ }^{\mathrm{a}}$, M. Balden ${ }^{\mathrm{a}}$, R.Dux ${ }^{\mathrm{a}}$, S. Elgeti ${ }^{\mathrm{a}}$, H. Gietl ${ }^{\mathrm{a}, \mathrm{b}}$, H. Greuner ${ }^{\mathrm{a}}$, A. Herrmann ${ }^{\mathrm{a}}$, T. Höschen ${ }^{\mathrm{a}}$, M. Li $^{\mathrm{a}}$, V. Rohde ${ }^{\mathrm{a}}$, \\ D. Ruprecht ${ }^{\mathrm{a}}$, B. Sieglin ${ }^{\mathrm{a}}$, I. Zammuto ${ }^{\mathrm{a}}$, ASDEX Upgrade Team ${ }^{\mathrm{a}}$ \\ ${ }^{a}$ Max-Planck-Institut für Plasmaphysik, 85748 Garching, Germany \\ ${ }^{b}$ Technische Universität München, Boltzmannstrasse 15, 85748 Garching, Germany
}

\begin{abstract}
Tungsten heavy alloy (97 wt.\% W, 2 wt.\% Ni, 1 wt.\% Fe) was investigated as an alternative for tungsten (W) as plasma facing material. It is produced commercially by several companies and compared to bulk W it is readily machinable and considerably cheaper. In order to qualify the material for use in the divertor of the mid-size tokamak ASDEX Upgrade (AUG) dedicated laboratory investigations as well as high heat flux tests in the neutral beam facility GLADIS were performed. These investigations revealed that the thermal conductivity at high temperature is close to that of $\mathrm{W}$, the magnetisation is small and saturates already at low magnetic field and the hydrogen retention is similarly low as that of W. In high heat flux tests at power densities up to 20 $\mathrm{MWm}^{-2}$ no failure was observed up to the melting temperature $\left(\approx 1500^{\circ} \mathrm{C}\right)$ of the binder phase. Even at surface temperatures of up to $2200{ }^{\circ} \mathrm{C}$ the mechanical integrity was sustained. Mechanical tests confirm the ductile behaviour of the W heavy alloy at room temperature and finite element analyses using the aforementioned data suggest a lower tendency for cracking. The increase of the long term dose-rate resulting from the activation of $\mathrm{Ni}$ under neutron irradiations appears to be moderate. During the 2017 campaign more than one fifth of the AUG divertor tiles consisted of W heavy alloy. Under nominal operation conditions the tiles showed no macroscopic failure and no increased Fe/Ni influx into the plasma was detected. Even though a few tiles showed strong melting at the edges due to accidental misalignment no failure due to cracking was observed.
\end{abstract}

Keywords: tungsten heavy alloy, tungsten, plasma-facing components, high heat flux testing, ASDEX Upgrade

\section{Introduction}

Since 2014 ASDEX Upgrade (AUG) is using bulk tungsten ${ }_{23}$ (W) tiles at the outer divertor strike-point. During this pe- 24 riod discharges with record values of injected power and en- 25 ergy were successfully performed. However, regular inspec- 26 tions between the campaigns revealed that a large number of ${ }_{27}$ tiles suffered from deep cracking attributed to brittle fracture ${ }_{28}$ [1]. Amongst different remedial actions [2], the use of W heavy 29 alloys (W-HAs) as a substitute for bulk tungsten was envisaged ${ }_{30}$ [3]. W-HAs are commercially available in different material ${ }_{31}$ compositions and due to their simpler production process they ${ }_{32}$ are considerably cheaper than $\mathrm{W}$. They consist mainly of $\mathrm{W}_{33}$ (> 90 wt.\%) with admixtures of $\mathrm{Ni}, \mathrm{Fe}$ or $\mathrm{Cu}$ (or other elements) ${ }_{34}$ in varying amounts. Due to their high density and strongly in- ${ }_{35}$ creased ductility in comparison to $\mathrm{W}$, they are used in industry (balancing weights), research (radiation shielding) and defense (penetrators). Only recently they were envisaged as a target material in a spallation source [4], which poses similar high heat ${ }^{37}$ load and fatigue requirements as in fusion applications.

Before the use in AUG, several investigations and tests on ${ }_{39}$ different W-HA were conducted (see [3]). Since erosion by plasma particles and release of alloying elements towards the plasma should be as small as possible, an independent boundary condition was set on minimizing the amount of alloying elements. As a consequence of these preparatory investigations and considerations W-HA with 97 wt.\% W, 2 wt.\% Ni, 1 wt.\% Fe was chosen for refined analysis and subsequent use in AUG.

In Sec. 2 earlier preparatory investigations and high heat flux tests are summarized and new results on mechanical properties and hydrogen retention are presented. Section 3 reports on the behaviour observed in the divertor of AUG and the microscopic analysis of W-HA tile surfaces at strongly overloaded leading edges. Finally, Sec. 4 concludes the paper and provides the outlook on possible applications of tungsten heavy alloys in fusion devices.

\section{Properties of Tungsten Heavy Alloys}

\subsection{Initial characterisation}

As a result of the preparatory investigations described in more detail in [3], W-HAs with 2 wt.\% Ni and 1 wt.\% Fe were 


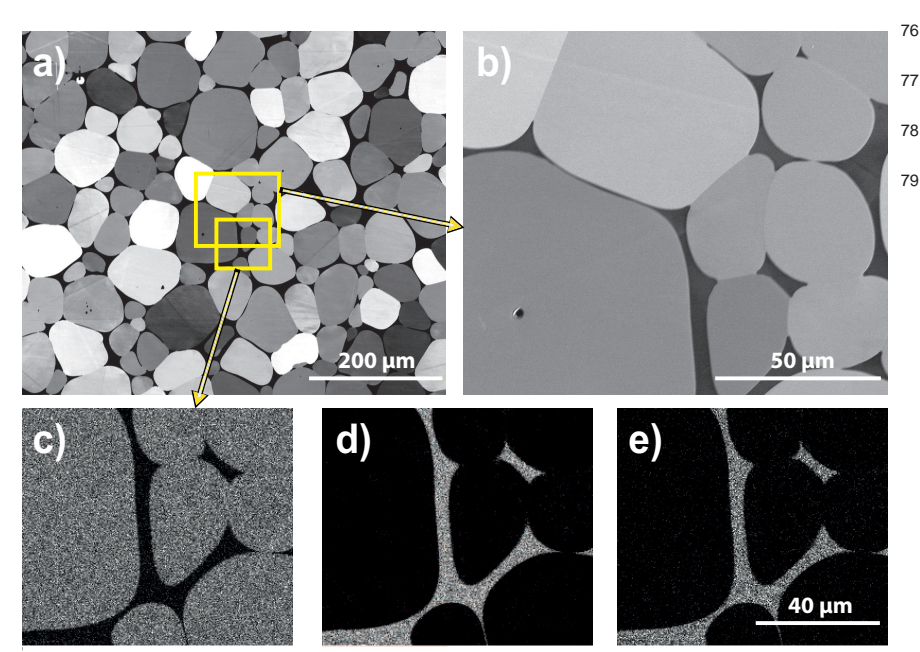

Figure 1: a) and b) SEM images of HPM 1850 with orientation contrast with different magnifications as well as EDX maps showing the distribution of c) W, d) $\mathrm{Ni}$ and e) Fe.

envisaged for use in AUG. They are available from many com- 70 mercial suppliers and are produced by pressing and sintering of powders or liquid phase sintering. The W-HA finally cho- 7 sen was HPM 1850 supplied by HC Starck Hermsdorf GmbH. D185 supplied by Plansee Composite Materials GmbH was also tested during the preparatory investigations and revealed similar properties as HPM 1850.

Figures 1a) and b) show typical Scanning Electron Microscope (SEM) images with orientation contrast of a polished 80 HPM 1850 surface. The rounded shape of the W grains is typ- 81 ical for W-HAs. In order to achieve highest densities different 82 powder sizes are mixed for the final product. Since during the 83 production process no cold work hardening is applied, the dif- 84 ferent grains appear to consist out of single crystals as can be 85 identified from the uniform shade of grey of the single grains 86 and as it could be confirmed in electron backscatter diffraction 87 measurements. As a consequence, one could expect that the 88 $\mathrm{W}$ matrix will not strongly change its properties when operated 89 close to the recrystallisation temperature. Figure 1b) presents a 90 close up of the picture. There it can be clearly seen that almost 91 all W grains are partly sintered (it can be expected that this will 92 be even more the case when taking into account all three dimen- 93 sions). This means that the material's properties will strongly 94 depend on the properties of the bonding of the $\mathrm{W}$ grains and the 95 properties of the $\mathrm{W}$ grains themselves and not just result from 96 the surrounding Ni-Fe phase (see also Fig. 4). Figures 1 (c)-d)) 97 present the elemental distribution measured by Energy Disper- 98 sive X-Ray Spectroscopy (EDX) of a detail of Fig. 1a). Within 99 the accuracy of the EDX measurement the grains consist only of $f_{100}$ $\mathrm{W}$ and the Ni-Fe phase shows the 2:1 composition as expected ${ }_{101}$ from weight percentages given by the manufacturer.

\subsection{Results of preparatory investigations}

The temperature dependent magnetisation and thermal conductivities of HPM1850 and D185 had been presented in [3]. The magnetisation of these materials is moderate $(\approx 2$ $\left.\mathrm{Am}^{-2} \mathrm{~kg}^{-1}\right)$ and saturates already at very low magnetic field $(<5000 \mathrm{Oe})$. The thermal conductivity at room temperature is a factor of two smaller than that of $\mathrm{W}\left(80 \mathrm{Wm}^{-1} \mathrm{~K}^{-1}\right)$, but in contrast to $\mathrm{W}$ it rather increases with $T$ leading to a similar thermal performance above $700{ }^{\circ} \mathrm{C}$.

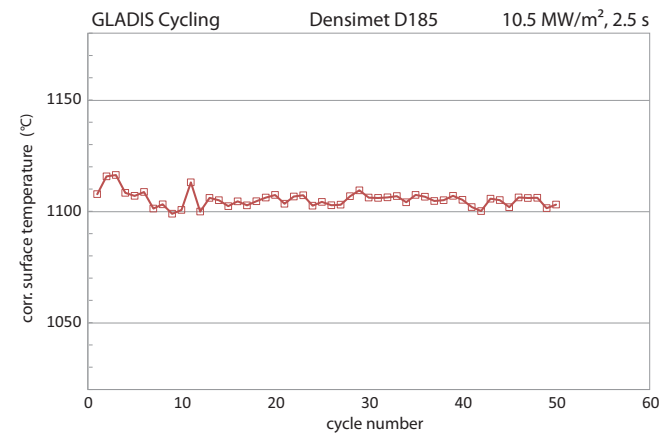

Figure 2: Evolution of the surface temperature of Densimet D185 (corrected for different starting temperatures) during thermal cycling evaluated at the end of each $10.5 \mathrm{MWm}^{-2} / 2.5 \mathrm{~s}$ pulse.

High heat flux (HHF) experiments were performed in the GLADIS facility [5], in order to test the full size W-HA divertor tiles under power loads expected during the operation of AUG. They consisted of

- thermal screening, where the adiabatically deposited power and energy was increased up to damage of the material in order to find the limit for the tolerable load and to investigate the material's behaviour under overload, and

- thermal cycling using a moderate power/energy load, similar to that expected in AUG in order to investigate the low cycle fatigue.

During the screening power loads of up to $20 \mathrm{MWm}^{-2}$ were applied and surface temperatures of up to $2200{ }^{\circ} \mathrm{C}$ were reached. This led to a segregation of $\mathrm{Ni}$ and $\mathrm{Fe}$ at the surface, but no mechanical failure was observed (for details see [3]). The cyclic loading was performed with 50 pulses at 10.5 $\mathrm{MWm}^{-2}$ with a pulse length of $2.5 \mathrm{~s}$, leading to typical surface temperatures in the range of $1100{ }^{\circ} \mathrm{C}$. Figure 2 presents the evolution of the surface temperature of D185 at the end of each pulse during the cycling. During the first phase of the cycling sequence the starting temperature of the target was rising because it is only clamped onto a water-cooled copper plate to 
simulate the adiabatically loading in ASDEX Upgrade. After a ${ }_{135}$ few cycles the start temperature becomes stable and in fig. 2 the ${ }_{136}$ measured surface temperature was corrected to a nominal start-137 ing temperature of $20{ }^{\circ} \mathrm{C}$. Taking this correction into account $\mathrm{a}_{138}$ very regular behaviour and only a minor scatter of the surface ${ }_{139}$ temperature is measured. After the cycling no macroscopic nor ${ }_{140}$ microscopic damage of the tiles was found. Since the divertor ${ }_{141}$ surface temperature in AUG stays well below $1100{ }^{\circ} \mathrm{C}$ in most ${ }_{142}$ of the discharges these results provided enough confidence to ${ }_{143}$ install the tiles in AUG.

\subsection{Mechanical behaviour}

In order to provide further input for the Finite Element analysis (see Sec. 3), the mechanical properties were measured after different heat treatments (for details see [6]). It turned out that tensile test specimen machined from as targets showed only a strain to rupture in the range of $0.5 \%$ at room temperature. However after treatment at elevated temperatures, elongations in the range of ten percent were achieved. An example for the investigations on HPM 1850 which was tempered at $1100^{\circ} \mathrm{C}$ for one hour is presented in Fig. 3. There the stress strain curves of several specimen are plotted. The tensile tests were performed with specimen (gauge length $12 \mathrm{~mm}$ ) proportional according to the standards in ASTM E8 and the loading speed was $10 \mu \mathrm{ms}^{-1}$. The strain was deduced contact-less from image evaluation in order to eliminate artefacts from the testing setup.

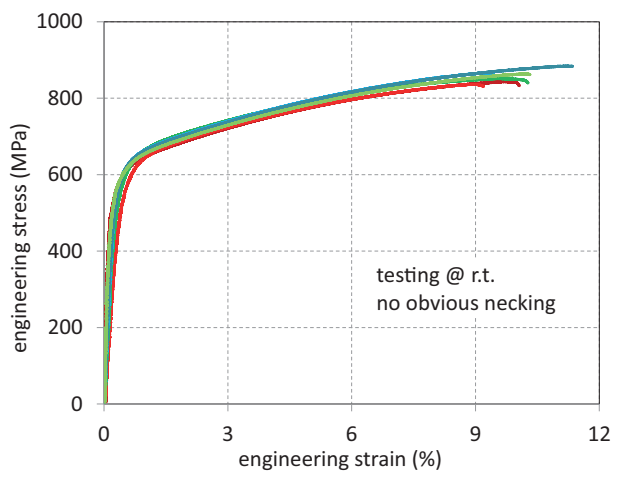

Figure 3: Stress - strain curves for HPM 1850 tempered at $1100{ }^{\circ} \mathrm{C}$ for 1 hour.

Remarkably, all the tested specimen show very similar yield 164 strength, ultimate tensile strength and elongation, which reflects 165 the ductile behaviour (in contrast to tungsten which typically 166 fails brittle at room temperature). However, no obvious necking ${ }_{167}$ was observed during plastic deformation. Another interesting ${ }_{168}$ feature discovered during the tensile tests is the fact that the 169 as-received samples show a very similar yield strength as the 170 tempered samples, however the region of plastic deformation is ${ }_{171}$ 149 150
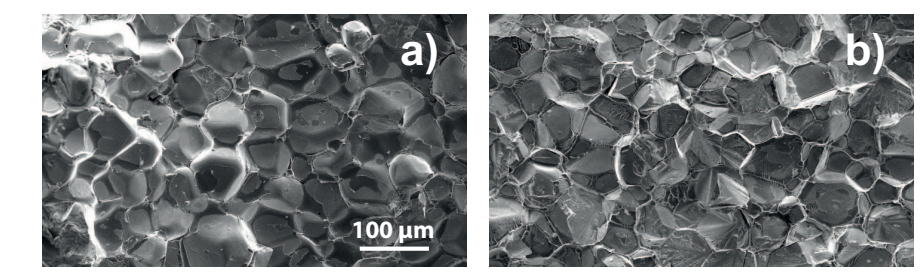

Figure 4: SEM images of the fracture surfaces of HPM 1850 a) as-received and b) after tempering at $1100{ }^{\circ} \mathrm{C}$ for 1 hour.

almost absent. For samples tempered at $1350{ }^{\circ} \mathrm{C}$ for one hour an even larger strain to rupture ( $>15 \%)$ was observed.

For comparison, additional tensile measurements were performed with $\mathrm{W}$ specimen cut from original ASDEX Upgrade tiles and treated in the similar way as the W heavy alloy specimen. The $\mathrm{W}$ specimen were cut in the direction of the highest thermal stresses (see Sec. 3.1) which is in the plain of, but perpendicular to the rolling direction. In total, five $\mathrm{W}$ specimen without thermal pre-treatment and five specimen tempered at $1100^{\circ} \mathrm{C}$ for 1 hour were tested. The ultimate tensile strength deduced from the valid tests was in the ranged of $340-540 \mathrm{MPa}$ in the case of the as-received specimen and $380-470 \mathrm{MPa}$ in case of the tempered ones. As expected for a brittle material, the elongation was very small ( $0.1 \%)$, and the ultimate tensile strength showed a large scatter but no influence of the tempering.

In order to gain insight into the improved tensile properties of the W-HA, the fracture surfaces were investigated by SEM. Figure 4 shows the fracture surface of HPM 1850 in its a) asreceived state and b) after tempering at $1100{ }^{\circ} \mathrm{C}$ for 1 hour. From the appearance and the shape of the surfaces one can conclude, that in the as-received state the fracture is mostly intergranular, whereas in the tempered state the adhesion of the grains appears to be much stronger and the final failure is mostly intragranular. Obviously, the tempering leads to a strengthening of the bonding at the grain boundaries without embrittling the material.

\subsection{Hydrogen retention}

Another important question for the use of a material in a fusion device is its interaction with hydrogen. A major advantage of W is its low hydrogen retention [7] which is seen as a prerequisite for its use in a fusion reactor. Although this property is not of predominant importance in present day devices, because they typically operate only with hydrogen and deuterium, the use of material with high hydrogen retention could strongly influence the overall hydrogen retention and eventually even the edge plasma and thereby hamper other plasma wall interaction 
investigations. In order to determine the hydrogen retention of 198 HPM 1850, samples were exposed together with W samples to 199 the low energy laboratory deuterium (D) plasma in the Garch-200 ing PlaQ device [8]. The targets were biased and the mean en-201 ergy of the impinging deuterium ions was $38 \mathrm{eV} / \mathrm{ion}$. The sam-202 ples were loaded with three different $\mathrm{D}$ fluences, namely with 203 $10^{23}, 10^{24}$ and $10^{25} \mathrm{Dm}^{-2}$ and kept at a temperature of $100^{\circ} \mathrm{C} .204$ After the loading, the samples were subjected to temperature 205 programmed desorption at a rate of $15 \mathrm{Kmin}^{-1}$. More details on 200 the exposure and the desorption can be found in [9]. Figure $5_{207}$

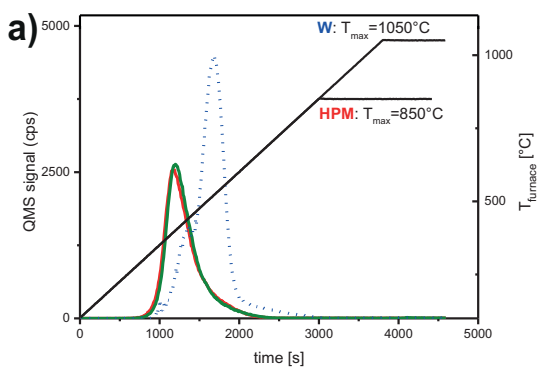

b)

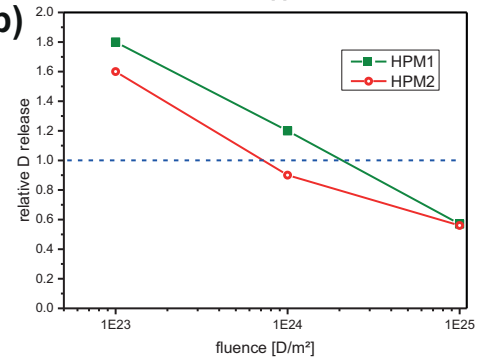

Figure 5: a) Thermal desorption spectra of HPM 1850 (solid lines) and $\mathrm{W}^{225}$ (dotted lines) after exposure to $10^{25} \mathrm{D} \mathrm{m}^{-2}$ (details see text). b) Ratio of D retention in HPM 1850 and $\mathrm{W}$ for three D fluences.

shows the results of the desorption measurements. The top part (5a)) shows the preprogrammed temperature which was ramped up to $850^{\circ} \mathrm{C}$ in the case of HPM 1850 and up to $1050^{\circ} \mathrm{C}$ in the case of $\mathrm{W}$, respectively (temperature scale at the right). The $\mathrm{D}$ desorption spectra are presented for the case of the highest fluence $\left(10^{25} \mathrm{D} \mathrm{m}^{-2}\right)$. As can be easily judged from the figure, the maximum of the desorption peak is at significantly lower temperature in the case of HPM $1850\left(\approx 400^{\circ} \mathrm{C}\right)$ compared to W $\left(\approx 550^{\circ} \mathrm{C}\right.$ ). For HPM 1850 there are two spectra shown (red, green) belonging to two different samples. The spectra overlay almost perfectly hinting to very homogeneous properties of the material and to the good reproducibility of the measurement. The integral under the desorption spectra seems to be smaller in the case of HPM 1850 compared to W. This is confirmed in 226 Fig. 5b) where the ratio of the retained D (integral below des-227 orption spectrum) of HPM 1850 to W is plotted for all three 228

fluences. For the highest fluence the retention in HPM 1850 is almost a factor of two lower than that of $\mathrm{W}$, whereas for low fluences the ratio is opposite. Currently, there is no clear explanation available, but most likely this observation is linked to different properties of the materials with respect to deuterium diffusion into the depth. The apparent diffusion coefficient of D in W depends very strongly on the density of trapping sites and the respective trapping energies. An increase of the trap density can lead to an increased retention and at the same time to less deep diffusion into the sample material. In addition the $\mathrm{Fe} / \mathrm{Ni}$ binder phase provides an additional very fast diffusion channel into the bulk. In principle one could expect a lower H-retention in W-heavy alloys, because the large grains consist of single crystal W (as mentioned in Sec. 2) with fewer defects than rolled tungsten. Nuclear reaction analysis investigations of the D depth profiles are ongoing and will be presented in a forthcoming paper [9]. However in the frame of this investigation it can be stated the the retention of both materials is similar and the desorption from HPM 1850 starts already at lower temperatures.

\subsection{Activation properties}

For a possible application in a fusion reactor, the transmutation and activation behaviour under neutron load must be taken into account. As a first step, the dose rate after 5 full power years in a DEMO reactor is derived from the data provided in [10]. Since the W-HA would be intended as a plasma facing material the activation data resulting from the neutron spectrum impinging the first wall were used.

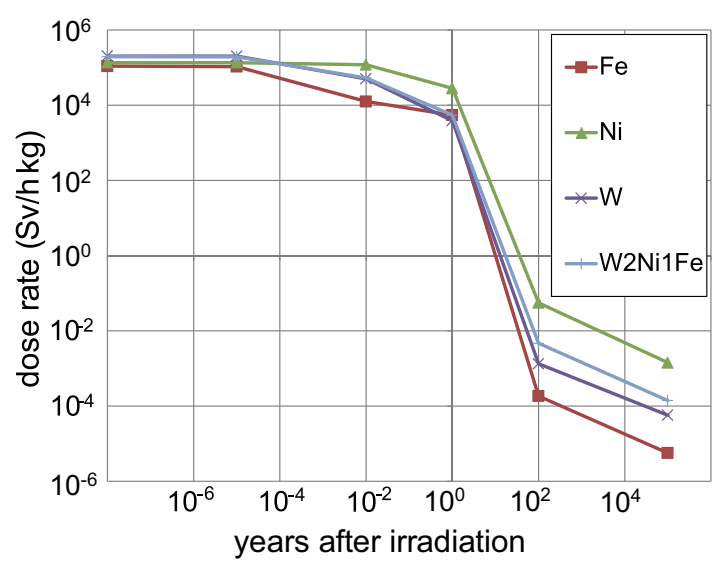

Figure 6: Calculated dose-rate (after 5 full power years in DEMO) from $\mathrm{W}, \mathrm{Ni}$, $\mathrm{Fe}$ and $\mathrm{W}$ heavy alloy with $2 \%$ and $1 \% \mathrm{Ni}$ and $\mathrm{Fe}$, respectively (calculated from data given in [10] (details see text).

Figure 6 presents the dose-rate per mass for the three constituents separately and the weighted sum for the W-HA with $2 \% \mathrm{Ni}$ and $1 \% \mathrm{Fe}$ (as for example HPM 1850). Because of its 
long-lived activation, which is about a factor of 10 higher than that of W, Ni should be avoided as a material. Conversely, the dose-rate from iron for similar n-irradiation is about a factor of 5 lower than that of W. As a consequence the W-HA shows very similar dose-rates as pure $\mathrm{W}$ up to a year after irradiation and less than a factor of two higher dose-rates after $10^{4}$ years. Although the longterm activation should be reduced as much as possible, this slightly higher dose-rate might be acceptable if other simpler technical solutions with W-HA were found which allow to reduce the overall amount of material (for example flattiles instead of monoblocks).

\section{Exposure in the ASDEX Upgrade Divertor}

\subsection{Finite Element analysis of stresses under thermal load 272}

Finite element analyses were performed for the AUG diver ${ }_{274}$ tor tiles (size: $15 \times 77 \times 227 \mathrm{~mm}^{3}$ ) consisting of $\mathrm{W}$ and $\mathrm{W}_{275}$ heavy alloy, respectively. The temperature-dependent material ${ }_{276}$ properties of W-HA were partly measured previously [3] and $_{277}$ partly taken from the literature [11]. Further details of the $\mathrm{FE}_{278}$ model can be found in [12]. Under the same thermal loading - 279 $15 \mathrm{MWm}^{-2}$ for $2.5 \mathrm{~s}$ - the maximum temperature of a W target ${ }_{280}$ reaches $1028^{\circ} \mathrm{C}$, and that of $\mathrm{W}$ heavy alloy target is at $1315^{\circ} \mathrm{C}_{28}$ Figure 7 shows the stress distribution of a W and a W-HA tile ${ }_{282}$ in toroidal direction, i.e. the stress component which is respon-283 sible for the opening of deep cracks in poloidal direction (verti- 284 cally). The stress field was taken after 5 thermal loading cycles $_{285}$ (2.5 s loading, $30 \mathrm{~s}$ dwell time) in the cooled-down state. The ${ }_{286}$ stress distribution in the two targets resulting from the different ${ }_{287}$ thermal conductance, modulus of elasticity and yield stress are $_{288}$ markedly different. The maximum stress appears at the top sur- ${ }_{289}$ face in the $\mathrm{W}$ target, while it is below the surface in the $\mathrm{W}-\mathrm{HA}_{290}$ target. The maximum stress in the $\mathrm{W}$ target is higher than $800_{291}$ $\mathrm{MPa}$, while it is about $500 \mathrm{MPa}$ in the $\mathrm{W}$ heavy alloy target.292 Compared to the $\mathrm{W}$ target, the W-HA has a larger area of stress ${ }_{293}$ concentration.

In the cases of $\mathrm{W}$, the calculated stress is higher than the mea-295 sured tensile strength of the material used in AUG (see Sec.296 2.3)) which hints to the fact that the observed cracks in $\mathrm{W}$ can 29 indeed be induced by the thermal stresses during target cool$_{298}$ down. Even when using $\mathrm{W}$ grades with higher tensile strength,299 brittle cracking due to thermo-mechanical fatigue, initial de-soo fects or other loading sources, would be more critical for $\mathrm{W}_{301}$ because its fracture toughness is lower than that of $\mathrm{W}$ heavy 302 alloy at room temperature [13, 14].

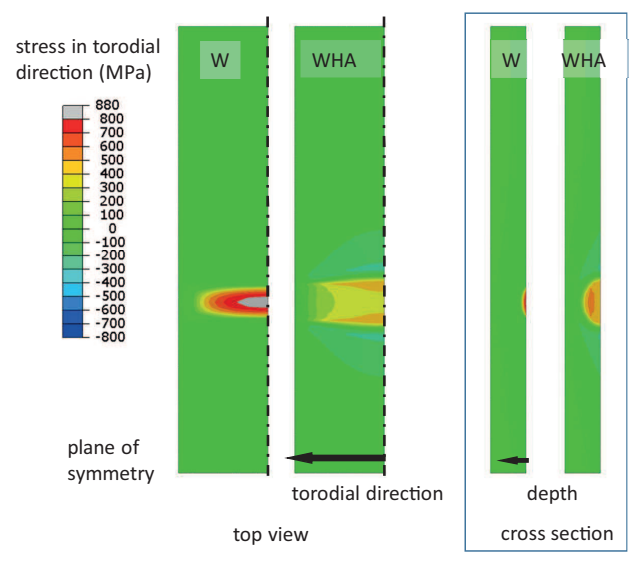

Figure 7: Stress distribution along the toroidal direction (only half of the tiles is plotted) as well as in the tile's cross section after 5 cycles of thermal loading (details see text).

\subsection{Performance of W-HA during the 2017 campaign}

As stated in the introduction, the installation of W-HA in the divertor was part of a strategy to prevent deep cracking of the divertor target tiles [2]. Within this strategy W-HA tiles were installed at the sector boundaries, since at these locations, the highest electro-mechanical loads due to halo currents were expected. Having 16 sectors in AUG, this means that there are 32 edge tiles. Since some of these edge tiles are specific tiles which were not exchanged presently, $28 \mathrm{~W}$-HA tiles out of a total of 128 divertor tiles were installed before the 2017 campaign. During 2017 almost 1000 plasma discharges were performed, about 40 of them had an auxiliary heating power of more than $15 \mathrm{MW}$ and several of them were heated with up to $20 \mathrm{MW}$ (see also Fig. 7 in [2] for more details). During many discharges one W-HA tile was monitored spectroscopically. Unfortunately, the sensitivity of the diagnostic was lower than expected. Nevertheless the (small) W influx from the tiles could be regularly detected but no Fe or Ni lines were evident, suggesting that the influx of $\mathrm{Fe}$ and $\mathrm{Ni}$ was as low as expected.

After six months of operation the machine was routinely opened during the summer break to repair minor defects of PFCs and diagnostic. Due to high halo currents during disruptions resulting in large moments and forces to the PFCs and their mounting, some of the divertor mountings were slightly twisted (see [1,2]) exposing the edges of the tiles at the sector boundary. Due to the almost perpendicular impact of the plasma, these edges receive about 10-20 times higher power load than the ordinary divertor surfaces, which easily leads to melting conditions for W-HA and W within seconds. Figure 8 shows the view into the divertor of AUG displaying three neighbouring tiles. As marked in the figure, the left and the right one consist out of tungsten, whereas the middle one is made 

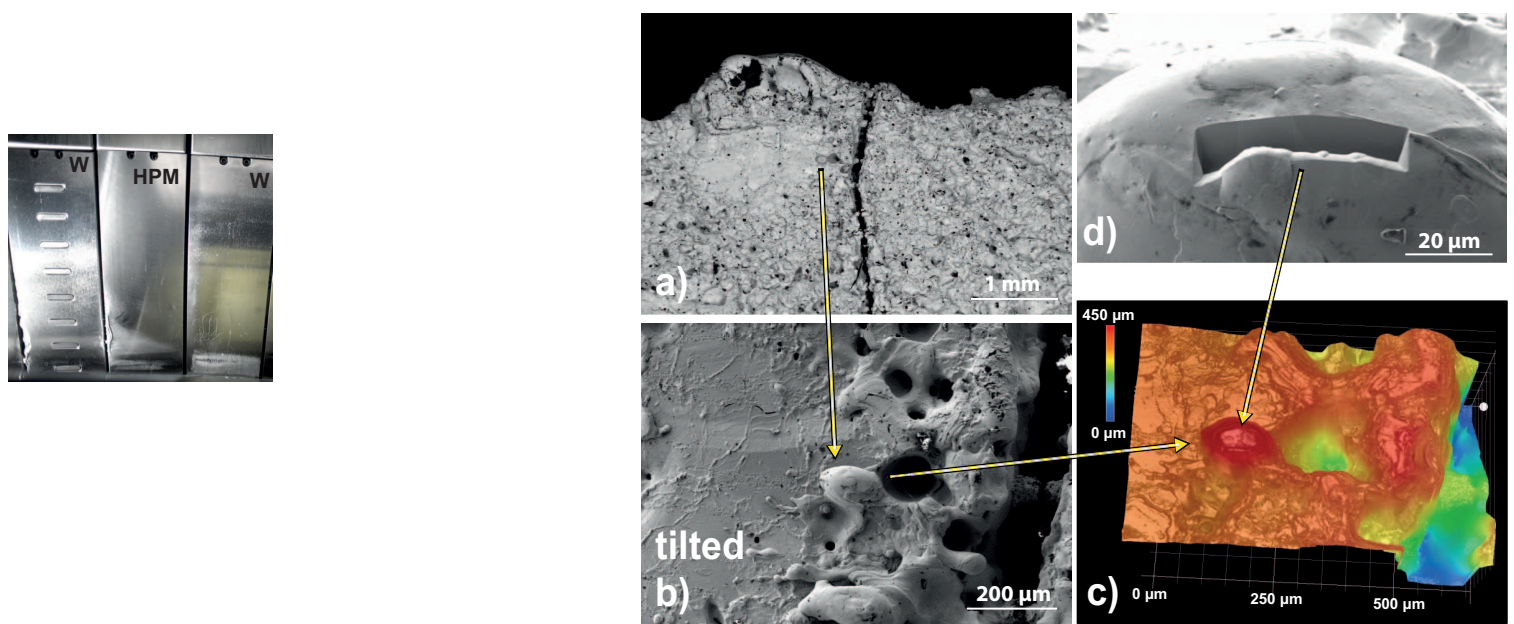

Figure 8: View into the divertor of ASDEX Upgrade after 6 months of operation. From left to right: W tiles with langmuir probes, HPM 1850 tile, W tile. ${ }_{331}$ The width of each tile is $77 \mathrm{~mm}$.

out of W-HA (HPM 1850). The right W tile is a specially in- ${ }_{334}$ strumented tile (shunt tile) and was therefore kept without any ${ }_{335}$ changes from the previous campaign. On this tile a distinct ${ }_{336}$ crack network developed in the strike-point area (lower third of the tile). As described in more detail in [2] this network consists of shallow and deep cracks some of the latter even reaching the ${ }_{339}$ back side of the tile. The sector boundary is between the left $\mathrm{W}_{340}$ tile (which is a equipped with flush-mounted Langmuir probes) and the W-HA tile. Due to an evolving misalignment (see ex- ${ }_{342}$ planation above) these two tiles were exposed to perpendicular plasma impact leading to heavy melting in the strikepoint re- ${ }_{344}$ gion. Although details on the exact power loads at the leading ${ }_{345}$ edges are not available, it appears that the region affected by ${ }_{346}$ melting looks very similar for both materials.

3.3. Microscopic loaded areas

-

During the short vent the molten tiles were retrieved and re-351 placed by new W and W-HA tiles, respectively, and the compo-352 nents were realigned to avoid further melting. The W-HA was353 investigated in detail with SEM and a Digital Microscope (DM)354 measuring the surface topography and the elemental composi-355 tion (see Figs. 9 and 10, respectively). Figure 9 presents details356 of the heavily molten area, revealing a grainy surface and cracks (Fig. 9a)). The surface is covered by melt noses and pools (Fig. ${ }_{357}$ 9b)) with vertical dimensions in the order of several hundred $\mu \mathrm{m}$, according to the DM measurements (Fig. 9c)). The Fo-358 cussed Ion Beam (FIB) cut in Fig. 9d) reveals that the top layer 359
Figure 9: SEM (a), b) and d)) micrographs and (c)) a DM topographical map with details of molten parts of a HPM 1850 tile from the divertor of AUG.

of the melt nose consists mostly of pure W. The structure and the elemental composition of the top surface layers further away from the fully molten area is presented in Fig. 10. In this area the temperature was below the melting temperature of $\mathrm{W}$ but above the melting temperature of $\mathrm{Ni}$ and $\mathrm{Fe}$. Due to a small vertical step at the crack which probably originates from the melt event, the area to the left of the crack seems to be erosion dominated and to the right deposition seems to prevail (Fig. 10a)). The small pores, clearly visible in the left part of the figure, represent areas where the $\mathrm{Ni} / \mathrm{Fe}$ filler is lost. Some of the missing material can be found as surface layer on top of the $\mathrm{W}$ grains (Figs. 10b) and c)). Interestingly, the loss of the filler materials does not necessarily lead to a loss of $\mathrm{W}$ grains, since their adhesion to other W grains (see Sec. 2) is obviously strong enough to keep the $\mathrm{W}$ skeleton intact. Since the $\mathrm{W}$ grains anyway were already recrystallized in the as-received state of the material, no strong evolution of the material's mechanical properties is expected, as also found in the tempering experiments described above and in more details in [6]. The surface in the 'deposition zone' (right part of Fig. 10a)) is covered by a thin layer of Ni/Fe (Fig. 10e)), and already a few $\mu \mathrm{m}$ in the depth of the tile Ni/Fe seems to be similarly distributed as in the as-received material (Fig. 10d)), in accordance with findings in the GLADIS HHF tests [3]. The latter is true also for the material on the left side of the crack as could be deduced from the FIB cuts also shown in Fig. 10a).

\section{Summary and Conclusions}

The investigated tungsten heavy alloys (W-HA) are composite materials with a large fraction of tungsten and a binder phase 


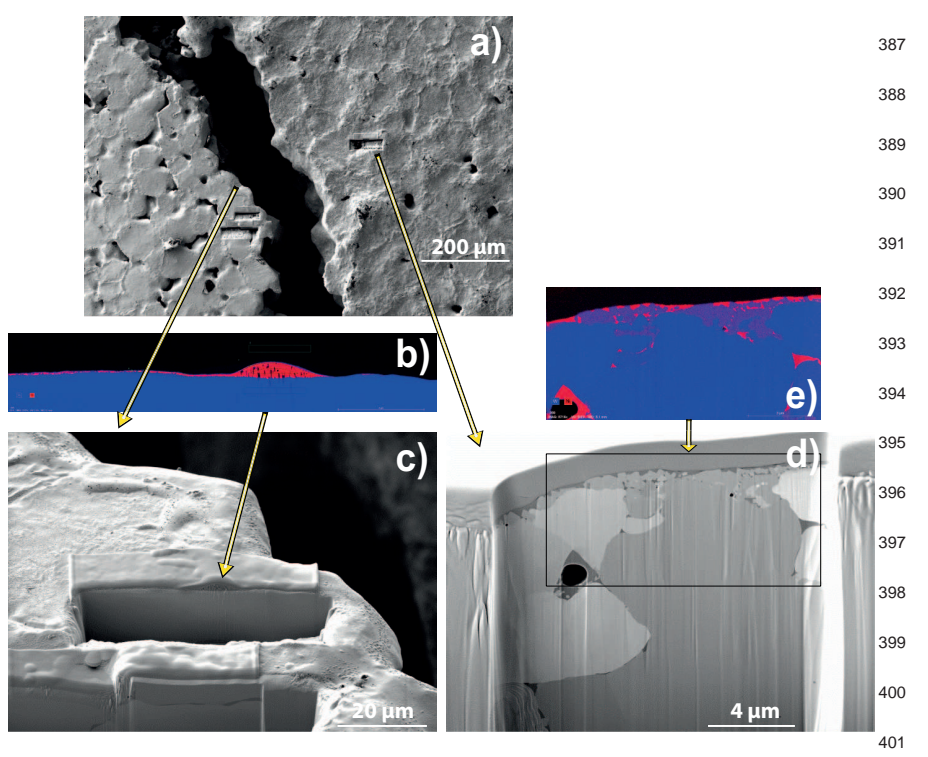

Figure 10. SEM micrographs and EDX maps on cross sections prepared FIB with details of overloaded parts of a HPM 1850 tile from the divertor of AUG. In the EDX maps, the blue colour represents W whereas the red areas ${ }^{404}$ predominantly consist out of $\mathrm{Ni}$ and $\mathrm{Fe}$.

\section{.}

During the routine operation of AUG the W-HA tiles neither show any specific behaviour nor was any increased $\mathrm{Fe} / \mathrm{Ni}$ influx or plasma content measured. Even under the accidental exposure of leading edges as a consequence of evolving misalignments at segment boundaries, the loss of the filler materials did not lead to a loss of $\mathrm{W}$ grains. Instead the $\mathrm{W}$ skeleton seemed to remain intact and when melting conditions for $\mathrm{W}$ were reached the W-HA tiles behaved very similar to their W neighbours.

Although the presented results are rather positive and justify the further use of W-HA in AUG, some remaining questions have to be answered before W-HA can be seriously considered for other future devices. One of them, mainly the long term behaviour under thermo-mechanical, electro-mechanical load as well as particle load will be further investigated in ASDEX Upgrade. If positive, W-HA may qualify as a practical surrogate for W PFCs in other mid-size devices like Wendelstein$7 \mathrm{X}$ or or the planned divertor test tokamak. Although the temperature range for the use of W-HA is restricted to temperatures below the melting temperatures of the filler material (i.e. $T_{\text {surf }}<1400^{\circ} \mathrm{C}$ ), this limit maybe relativised in view of the fact that $\mathrm{W}$ starts to recrystallise around $1300{ }^{\circ} \mathrm{C}$ and recent investigations (see [15]) suggest that the operational temperature for tungsten should kept below this value to preserve its original mechanical properties. For an application in a future reactor, dedicated investigations on the evolution of the hydrogen retention and the mechanical properties (i.e. embrittlement) under neutron irradiation need to be performed in order to confirm the rather advantageous properties.

\section{Acknowledgement}

The authors want to thank G. Matern for helping with the metallographic preparation of the samples and B. Böswirth for 
assisting the GLADIS tests. This work has been carried out within the framework of the EUROfusion Consortium and has received funding from the Euratom research and training programme 2014-2018 under grant agreement No 633053. The views and opinions expressed herein do not necessarily reflect those of the European Commission.

\section{References}

[1] A. Herrmann, I. Zammuto, M. Balden, H. Greuner, N. Jaksic, A. Kallenbach, M. Li, R. Neu, V. Rohde, and ASDEX Upgrade Team. Nuclear Materials and Energy, 12:205-209, 2017.

[2] I. Zammuto, M. Li, A. Herrmann, N. Jaksic, V. Rohde, M. Balden, S. Vorbrugg, G. Matern, H. Greuner, R. Neu, A. Kallenbach, and ASDEX Upgrade Team. Crack avoidance with a modified solid tungsten divertor in ASDEX Upgrade. submitted to Fus. Eng. Des., 2018.

[3] R. Neu, H. Maier, M. Balden, S. Elgeti, H. Gietl, H. Greuner, A. Herrmann, A. Houben, V. Rohde, B. Sieglin, I. Zammuto, and ASDEX Upgrade Team. Fusion Engineering and Design, 124:450-454, 2017.

[4] M. Pasalic, F. Rustempasic, S. Iyengar, S. Melin, and E. Noah. International Journal of Refractory Metals and Hard Materials, 42:163 - 168, 2014.

[5] H. Greuner, B. Boeswirth, J. Boscary, and P. McNeely. Journal of Nuclear Materials, 367370, Part B:1444 - 1448, 2007.

[6] M. Li et al. Impact of heat treatment on microstructure and mechanical properties of 97W-2Ni-1Fe. in preparation, 2018.

[7] Joachim Roth, E. Tsitrone, A. Loarte, Th. Loarer, G. Counsell, R. Neu, V. Philipps, S. Brezinsek, M. Lehnen, P. Coad, Ch. Grisolia, K. Schmid, K. Krieger, A. Kallenbach, B. Lipschultz, R. Doerner, R. Causey, V. Alimov, W. Shu, O. Ogorodnikova, A. Kirschner, G. Federici, and A. Kukushkin. Journal of Nuclear Materials, 390391:1 - 9, 2009.

[8] A Manhard, T Schwarz-Selinger, and W Jacob. Quantification of the deuterium ion fluxes from a plasma source. Plasma Sources Science and Technology, 20(1):015010, 2011.

[9] H. Maier, T. Schwarz Selinger, C. Garcia-Rosales, R. Neu, M. Balden, A. Calvo, T. Dürbeck, A. Manhard, N. Ordás, and T.F. Silva. Deuterium retention in tungsten-based materials for fusion applications. to be presented at the $23 r d$ Int. Conf on Plasma Surface Interaction (PSI23), Princeton, 2018.

[10] R.A. Forrest, A. Tabasso, C. Danani, S. Jakhar, and A.K. Shaw. Handbook of activation data calculated using easy-2007. In UKAEA FUS 552, Culham Science Centre, Abingdon, Oxfordshire, OX14 3DB, United Kingdom, 2009. EURATOM/UKAEA Fusion Association.

[11] S.H. Islam. Rare Metals, 30:392, 2011.

[12] M. Li, I. Zammuto, N. Jaksic, B. Boeswirth, H. Greuner, A. Herrmann, R. Neu, V. Rohde, J.-H. You, and ASDEX Upgrade Team. Numerical design for avoiding deep cracks in the solid tungsten divertor target in ASDEX Upgrade. submitted to Fus. Eng. Design, 2018.

[13] K.M.O. Zamara, J.G. Sevillano, and M.F. Pérez. Mater. Sci. Eng. A, 157:151-160, 1992.

[14] C.H. Henager, W. Setyawan, T.J. Roosendaal, N.R. Overman, B.A. Borlaug, E.L. Stevens, K.B. Wagner, R.J. Kurtz, G.R. Odette, B.N. Nguyen, and K.H. Cunningham. Ductile-phase toughened tungsten for plasmafacing materials in fusion reactors. Int. Jour. of Powder Metallurgy, 53:53 $-69,2017$.

[15] S. Panayotis, T. Hirai, V. Barabash, C. Amzallag, F. Escourbiac, A. Durocher, V. Komarov, J.M. Martinez, and M. Merola. Fusion Engineering and Design, 125:256 - 262, 2017. 